\section{A generalized approach for selecting solar energy system configurations for a wide range of applications - ADDENDUM}

doi: https://doi.org/10.1557/mre.2019.10

The following footnote should be included in this article [1]:

This paper was commissioned for publication by David Cahen, who served as Editor-in-Chief of this journal from 2014-2018.
Pinchas Doron, Jacob Karni, and Alexander Slocum

REFERENCE:

1. Doron P., Karni J., \& Slocum A. (2019). A generalized approach for selecting solar energy system configurations for a wide range of applications. MRS Energy \& Sustainability, 6, E11. Cambridge University Press. 